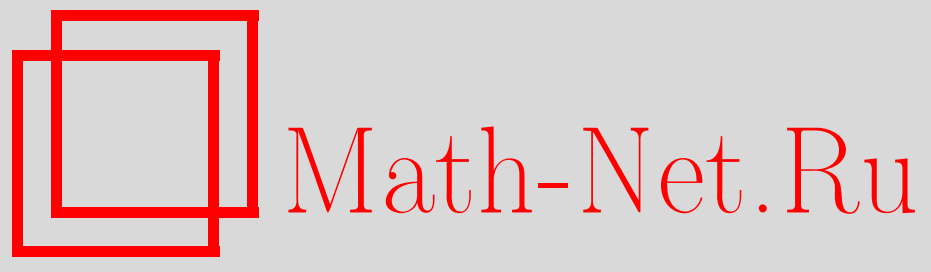

Ю. А. Кузнецов, Различение гипотез в непрерывной схеме с альтернативными направлениями, УМН, 2008, том 63, выпуск 2, 173-174

DOI: https://doi.org/10.4213/rm9190

Использование Общероссийского математического портала Math-Net.Ru подразумевает, что вы прочитали и согласны с пользовательским соглашением http://www . mathnet.ru/rus/agreement

Параметры загрузки:

IP : 3.80 .181 .102

26 апреля 2023 г., 14:38:40

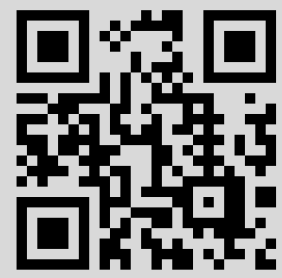




\section{Различение гипотез в непрерывной схеме с альтернативными направлениями}

\section{Ю. А. Кузнецов}

Пусть в каждый момент времени $t$ имеется возможность наблюдать или процесс $X^{1}=\left(X_{t}^{1}\right)_{t \geqslant 0}$ с $d X_{t}^{1}=I(\theta=1) d t+W_{t}^{1}$, или процесс $X^{2}=\left(X_{t}^{2}\right)_{t \geqslant 0}$ с $d X_{t}^{2}=$ $I(\theta=2) d t+W_{t}^{2}$. Здесь $W^{1}=\left(W_{t}^{1}\right)_{t \geqslant 0}$ и $W^{2}=\left(W_{t}^{2}\right)_{t \geqslant 0}$ - два независимых броуновских движения. Случайная величина $\theta$ принимает одно из трех значений 0,1 или 2. При этом событие $\theta=0$ интерпретируется как отсутствие “цели" на первом и втором направлениях. Событие $\theta=i$ означает наличие "цели" на $i$-м направлении $(i=1,2)$. Пусть $T_{i}(t)$ - общее время наблюдения $i$-го направления $(i=1,2)$. При этом $T_{1}(t)+T_{2}(t)=t$, где $t$ - общее время наблюдения. Задача состоит в нахождении $\left(T_{1}(t), T_{2}(t)\right)$ (управления), момента остановки $\tau$ и окончательного решения $d$ относительно $\theta$, при которых достигает минимума функиия риска

$$
R(s)=\mathrm{E}\left(c \tau+\alpha_{1} I(\theta=1,2 ; d=0)+\alpha_{2} I(\theta=0 ; d=1)\right),
$$

где $s=\left(T_{1}(t), T_{2}(t), \tau, d\right)$ - допустимая стратегия (допустимой стратегией называется такая четверка $\left(T_{1}(t), T_{2}(t), \tau, d\right)$, что выполнены следующие условия: $T_{1}(0)=$ $T_{2}(0)=0, T_{1}(t)+T_{2}(t)=t, T_{1}(t), T_{2}(t)$ - неубывающие функции, $\left\{T_{1}(t) \leqslant t_{1}\right.$,

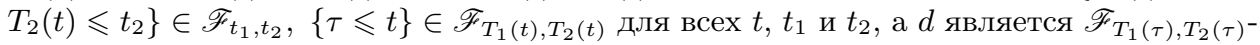
измеримой, где $\left.\mathscr{F}_{t_{1}, t_{2}}=\sigma\left(W_{u_{1}}^{1}, W_{u_{2}}^{2}, u_{1} \in\left[0, t_{1}\right], u_{2} \in\left[0, t_{2}\right]\right)\right)$. Здесь $\alpha_{i}-$ платы за принятие неправильных решений $(i=1,2)$ и $c$ - плата за наблюдения.

Задача впервые была поставлена и изучалась в [1].

Далее будем считать, что $\alpha_{1}=\alpha_{2}=1$. Положим $\pi_{i}(t)=\mathrm{P}\left(\theta=i \mid \mathscr{F}_{T_{1}(t), T_{2}(t)}\right)$, $i=0,1,2$.

Поскольку при остановке в момент $\tau$ окончательное решение $d$ определяется однозначно в зависимости от $\pi_{0}(\tau)$, то задача эквивалентна минимизации функции риска

$$
R(s)=\mathrm{E}\left(c \tau+\min \left(\pi_{0}(d), 1-\pi_{0}(d)\right)\right),
$$

где $s=\left(T_{1}(t), T_{2}(t), \tau\right)$. Минимальный риск обозначим через $\rho\left(\pi_{1}, \pi_{2}\right)$.

ОпредЕлениЕ. Управление $\left(T_{1}(t), T_{2}(t)\right)$ называется стандартным, если происходит наблюдение процесса $X_{t}^{1}$ (первого направления), когда $\varphi_{1}(t)>\varphi_{2}(t)$, и процесса $X_{t}^{2}$ (второго направления), когда $\varphi_{2}(t)>\varphi_{1}(t)$, где $\varphi_{i}(t)=\pi_{i}(t) / \pi_{0}(t), i=1,2$.

Теорема 1. В классе марковских стандартных стратегий оптималъное правило остановки определяется как момент первого попадания вектора $\left(\varphi_{1}(t), \varphi_{2}(t)\right)$ в некоторую область "остановки наблюдений" $(A \cup D$ на рис. 1). Область продолжения наблюдений разбивается на области возможных переключений между направлениями $\left(C_{1} \cup C_{2}\right)$ и область, где возможно наблюдение лишь одного направления $\left(B_{1} \cup B_{2}\right)$.

Найдем границы области продолжения наблюдения в той части плоскости, где наблюдается только одно направление.

Теорема 2. Границы $\gamma_{1}$ и $\gamma_{2}$ вместе с неизвестной функиией риска $\rho\left(\varphi_{1}, \varphi_{2}\right)$ в области $B_{1}$ могут быть найдены из системь

$$
\left\{\begin{array}{l}
\rho_{\varphi_{2}}^{\prime}\left(\varphi_{1}, \varphi_{2}\right) \varphi_{2}^{2} /\left(1+\varphi_{1}+\varphi_{2}\right)+(1 / 2) \rho_{\varphi_{2} \varphi_{2}}^{\prime \prime}\left(\varphi_{1}, \varphi_{2}\right) \varphi_{2}^{2}=-c \\
\rho\left(\varphi_{1}, \gamma_{1}\left(\varphi_{1}\right)\right)=\rho_{0}\left(\varphi_{1}, \gamma_{1}\left(\varphi_{1}\right)\right) \\
\rho\left(\varphi_{1}, \gamma_{2}\left(\varphi_{1}\right)\right)=\rho_{0}\left(\varphi_{1}, \gamma_{2}\left(\varphi_{1}\right)\right) \\
\rho_{\varphi_{2}}^{\prime}\left(\varphi_{1}, \gamma_{1}\left(\varphi_{1}\right)\right)=\left(\rho_{0}\right)_{\varphi_{2}}^{\prime}\left(\varphi_{1}, \gamma_{1}\left(\varphi_{1}\right)\right) \\
\rho_{\varphi_{2}}^{\prime}\left(\varphi_{1}, \gamma_{2}\left(\varphi_{1}\right)\right)=\left(\rho_{0}\right)_{\varphi_{2}}^{\prime}\left(\varphi_{1}, \gamma_{2}\left(\varphi_{1}\right)\right)
\end{array}\right.
$$

где $\rho_{0}\left(\varphi_{1}, \varphi_{2}\right)=\min \left(\pi_{0}, 1-\pi_{0}\right)=\min \left(\left(\varphi_{1}+\varphi_{2}\right) /\left(1+\varphi_{1}+\varphi_{2}\right), 1 /\left(1+\varphi_{1}+\varphi_{2}\right)\right)$. 


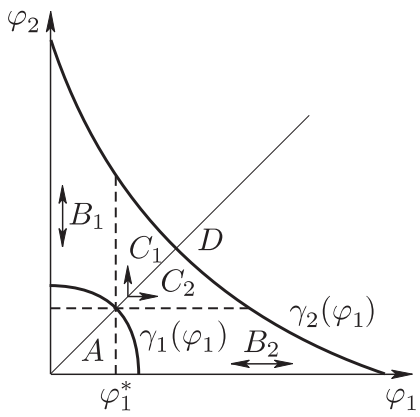

Рис. 1

Решения $\gamma_{1}\left(\varphi_{1}\right)$ и $\gamma_{2}\left(\varphi_{1}\right)$ этой системы могут быть получены следующим образом. Для каждого фиксированного $k=1+\varphi_{1}$ относительно $A^{*}$ и $B^{*}$ решаем систему

$$
\left\{\begin{array}{l}
2=C\left(\psi\left(B^{*}\right)-\psi\left(A^{*}\right)\right), \\
C\left(\Psi\left(B^{*}\right)-\Psi\left(A^{*}\right)\right)-C\left(B^{*}-A^{*}\right) \psi\left(A^{*}\right)=2-2 B^{*}-k,
\end{array}\right.
$$

при этом $C=2 k c, \Psi(\pi)=(1-2 \pi) \ln (\pi /(1-\pi)), \psi(\pi)=\Psi^{\prime}(\pi)$, и по полученным $A^{*}$ и $B^{*}$ находятся границы $\gamma_{1}\left(\varphi_{1}\right)=\left(A^{*} /\left(1-A^{*}\right)\right)\left(1+\varphi_{1}\right), \gamma_{2}\left(\varphi_{1}\right)=\left(B^{*} /\left(1-B^{*}\right)\right)\left(1+\varphi_{1}\right)$.

Заметим, что полученная система с $A^{*}$ и $B^{*}$ аналогична системе в одномерной задаче распознавания гипотез [2] и полностью совпадает с ней при $k=1\left(\varphi_{1}=0\right)$. Этот факт является следствием того, что в области, где возможно наблюдение одного направления, задача становится одномерной, а значит, для границ получаются уравнения, аналогичные приведенным в [2].

Теорема 3. Граница $\gamma_{2}$, разделяющая область остановки и область продолжения наблюдений в области $C_{1}$, задается с помощью кривой $\gamma_{2}=\gamma_{2}\left(\varphi_{1}\right)$, которая может быть найдена вместе с функиией риска $\rho\left(\varphi_{1}, \varphi_{2}\right)$ из системы уравнений

$$
\left\{\begin{array}{l}
\rho_{\varphi_{2}}^{\prime}\left(\varphi_{1}, \varphi_{2}\right) \varphi_{2}^{2} /\left(1+\varphi_{1}+\varphi_{2}\right)+(1 / 2) \rho_{\varphi_{2} \varphi_{2}}^{\prime \prime}\left(\varphi_{1}, \varphi_{2}\right) \varphi_{2}^{2}=-c \\
\rho_{\varphi_{1}}^{\prime}\left(\varphi_{1}, \varphi_{1}\right)=\rho_{\varphi_{2}}^{\prime}\left(\varphi_{1}, \varphi_{1}\right) \\
\rho\left(\varphi_{1}, \gamma_{2}\left(\varphi_{1}\right)\right)=\rho_{0}\left(\varphi_{1}, \gamma_{2}\left(\varphi_{1}\right)\right) \\
\rho_{\varphi_{2}}^{\prime}\left(\varphi_{1}, \gamma_{2}\left(\varphi_{1}\right)\right)=\left(\rho_{0}\right)_{\varphi_{2}}^{\prime}\left(\varphi_{1}, \gamma_{2}\left(\varphi_{1}\right)\right)
\end{array}\right.
$$

где $\rho_{0}\left(\varphi_{1}, \varphi_{2}\right)=\min \left(\pi_{0}, 1-\pi_{0}\right)=\min \left(\left(\varphi_{1}+\varphi_{2}\right) /\left(1+\varphi_{1}+\varphi_{2}\right), 1 /\left(1+\varphi_{1}+\varphi_{2}\right)\right)$.

Решая эту систему, получаем для границы $\tilde{\gamma}_{2}=\tilde{\gamma}_{2}\left(\varphi_{1}\right)$ (области $C_{1}$ ) дифференциальное уравнение

$$
\frac{\tilde{\gamma}_{2}^{\prime}}{\tilde{\gamma}_{2}^{2}}=\frac{\left(1 / \varphi_{1}-2 \ln \varphi_{1}\right)-\left(1 / \tilde{\gamma}_{2}-2 \ln \tilde{\gamma}_{2}\right)}{\left(\varphi_{1}-\tilde{\gamma}_{2}\right)\left(1+\varphi_{1}+\tilde{\gamma}_{2}\right)}
$$

с начальным условием $\tilde{\gamma}_{2}\left(\varphi^{*}\right)=\gamma_{2}\left(\varphi^{*}\right)$, где $\varphi^{*}$ таково, что $\gamma_{1}\left(\varphi^{*}\right)=\varphi^{*}$.

Автор благодарит А.Н. Ширяева за постановку задачи и полезные обсуждения.

\section{Список литературы}

[1] А. Н. Ширяев, Trans. 3rd Prague Conf. Information Theory, Statist. Decision Functions, Random Processes (Liblice, 1962), Czechoslovak Acad. Sci., Prague, 1964, 657-681. [2] А. Н. Ширяев, Статистический последовательный анализ, Наука, М., 1976.

Ю. А. Кузнецов (Yu. А. Kuznetsov)

Московский государственный университет им. М. В. Ломоносова

E-mail: yu_ku77@mail.ru
Представлено А. В. Булинским Принято редколлегией 17.02.2008 